\title{
FRAMEWORK FOR ONLINE SUPERIMPOSED EVENT DETECTION BY SEQUENTIAL MONTE CARLO METHODS
}

\author{
O. Urfalıog̃lu ${ }^{1}$ Ercan E. Kuruog̃lu ${ }^{2}$ and A. Enis Çetin ${ }^{1}$ \\ ${ }^{1}$ Department of Electrical and Electronics Engineering \\ Bilkent University, Ankara/Turkey \\ ${ }^{2}$ ISTI/CNR, Pisa/Italy
}

\begin{abstract}
In this paper, we consider online seperation and detection of superimposed events by applying particle filtering. We concentrate on a model where a background process, represented by a $1 \mathrm{D}$-signal, is superimposed by an Auto-Regressive (AR) 'event signal', but the proposed approach is applicable in a more general setting. The activation and deactivation times of the event-signal are assumed to be unknown. We solve the online detection problem of this superpositional event by extending the state space dimension by one. The additional parameter of the state represents the AR-signal, which is zero when deactivated. Numerical experiments demonstrate the effectiveness of our approach.
\end{abstract}

Index Terms - Event detection, Conditional Density, SIR, Importace Sampling, Bayesian Statistics

\section{INTRODUCTION}

Event detection is becoming an important and more frequently studied field in recent times. There are applications in intrusion detection, internet traffic analysis, bioinformation processing, telecommunication, surveillance and more. In this paper, online model based event detection using sequential Monte Carlo methods, namely particle filtering $[3,5,6,8,4,2]$, is studied. The term model based emphasizes that the stochastic model of the event is known. On the other hand, the activation time of the event is unknown and the event is superpositional with respect to a background process. This stochastic event-process is modeled as an Auto Regressive (AR) process, which superimposes a background stochastic process. So, in this setting, only the result of this superposition is observable. The task of the proposed approach is to simulate and estimate the hidden background process, to detect the event activation/deactivation times and to estimate also the hidden event process.

In many event detection methods [11], the estimated state or a sequence of estimated states is undergone a secondary analysis by e.g. using a Hidden Markow Model (HMM). This

This work was carried out during the tenure of a MUSCLE Internal fellowship.
HMM represents the model of the event statistics. MCMCbased methods as in $[9,7,10]$ are generally not applicable in an online approach due to high computational requirements. In [1], an overview of change point detection using particle filters is given. However, our approach not just attacks a change point detection problem, but enables also online source seperation.

In the proposed approach, the detection method of the event is embedded in the particle filtering framework directly. By increasing the state space dimension by the number of additional event process parameters and appropriately choosing the importance functions, we are able to estimate both the hidden process and the superpositional event process simultaneously. This is accomplished by minimal modification of the particle filtering framework.

\section{SEQUENTIAL MONTE CARLO METHODS - SIR}

In the Sequential Monte Carlo (SMC) setting, the stochastic process consists of hidden state propagation and observation, represented as a $\operatorname{HMM}(1)$. The state propagation $x_{t} \rightarrow x_{t+1}$ at time $t$ is modeled as

$$
x_{t+1}=f\left(x_{t}\right)+v_{t},
$$

and the observation $y_{t}$ is modeled as

$$
y_{t}=g\left(x_{t}\right)+w_{t}
$$

where $v_{t}$ and $w_{t}$ are independent random variables. The sequential Bayesian inference consists of a prediction step and an update step via

$$
\begin{aligned}
p\left(x_{t+1} \mid y_{1: t}\right) & =\int p\left(x_{t+1} \mid x_{t}\right) p\left(x_{t} \mid y_{1: t}\right) d x_{t} \\
p\left(x_{t+1} \mid y_{1: t+1}\right) & =\frac{p\left(y_{t+1} \mid x_{t+1}\right) p\left(x_{t+1} \mid y_{1: t}\right)}{p\left(y_{t+1} \mid y_{1: t}\right)}
\end{aligned}
$$

with

$$
y_{1: t} \stackrel{\wedge}{=}\left\{y_{1}, \ldots, y_{t}\right\}
$$


Due to generally intractable integrals, the sequential Bayesian inference is realized by approximation methods such as Sequential Importace Resampling (SIR) [3]. In the SIR framework, the posterior $p\left(x_{t} \mid y_{1: t}\right)$ is represented by a particle set of $N$ particles. A particle consists of a position vector $x_{n, t}$ and a weight-scalar $\omega_{n, t}$ with the approximation property

$$
p\left(d x_{t} \mid y_{1: t}\right) \approx \sum_{n=1}^{N} \omega_{n, t} \delta\left(d x_{n, t}\right),
$$

where

$$
\sum_{n=1}^{N} \omega_{n, t}=1
$$

The particle positions are sampled from an importance density

$$
x_{n, t+1} \sim \pi\left(x_{t+1} \mid x_{n, t}, y_{1: t+1}\right)
$$

at each time step. The weights are determined by

$$
\tilde{\omega}_{n, t+1}=\omega_{n, t} \frac{p\left(y_{t+1} \mid x_{t+1}\right) p\left(x_{t+1} \mid x_{t}\right)}{\pi\left(x_{t+1} \mid x_{n, t}, y_{1: t+1}\right)}
$$

and normalized afterwards

$$
\omega_{n, t+1}=\frac{\tilde{\omega}_{n, t+1}}{\sum_{n=1}^{N} \tilde{\omega}_{n, t+1}} .
$$

Due to degeneracy in this method regarding the importance weights, on which all but one particle has a weight of 1 and all others have zero weight, a resampling step is added after each iteration. The resampling is done by copying the particle $N \omega$ times in average by overwriting other particles, so particles with strong weights are reproduced more often, in average.

\section{FRAMEWORK FOR SUPERIMPOSED EVENT DETECTION}

The type of events we consider can be modeled as follows. The background signal, denoted by $x_{t}$, is superimposed by a second signal, denoted by $z_{t}$, which is independent of $x_{t}$

$$
x_{t+1}=f\left(x_{t}\right)+v_{t}+\alpha_{t} z_{t+1} .
$$

The event signal is assumed to be only present for some time window $T_{E}$

$$
\alpha_{t}=\left\{\begin{array}{cc}
1 & t \in T_{E} \\
0 & \text { else }
\end{array}\right.
$$

Since there is no 'pure' observation available from the signal $z_{t}$, it can only be estimated together with $x_{t}$. We assume that a parameteric description of the signal $z_{t}$, specified by

$$
z_{t+1}=h\left(z_{t}\right)+u_{t},
$$

is available

$$
z_{t}=z_{t}\left(\theta_{t}\right)
$$

The task is to detect the event, in this case to tell whether there is a superpositional $z_{t}$ present and to estimate $z_{t}$. The proposed approach consists of using an SIR-particle filter, whose state space dimension is extended by the number of the required additional hidden parameters, having the state vector $s_{t}$

$$
s_{t}=\left(x_{t}, z_{t}, \alpha_{t}, \theta_{t}\right) .
$$

Alternatively, the parameter $\alpha_{t}$ can be discarded by adapting the conditional probability density $p\left(z_{t+1} \mid z_{t}\right)$ of $z_{t}$ by

$$
p^{\prime}\left(z_{t+1} \mid z_{t}\right)=\frac{1}{2}\left(\delta(0)+p\left(z_{t+1} \mid z_{t}\right)\right)
$$

where $\delta($.$) is the Dirac substitution and \delta(0)$ produces exact zeros as 'no-event' samples.

The state propagation density for the superimposed signal can be written as

$$
p\left(x_{t+1}, z_{t+1} \mid x_{t}, z_{t}\right)=p\left(x_{t+1} \mid x_{t}\right) p\left(z_{t+1} \mid z_{t}\right) .
$$

\subsection{The choice of importance functions}

The choice of the importance function is crucial in the SIRframework, since it has a great impact on the efficiency and even feasability of the simulations. One of the most common methods is to use the state propagation density as the importance density function, as in [6]. Though this choice does not take the current observation into account, it is sufficient for many simulation problems.

The importance function for $\alpha_{t}$ can be chosen as

$$
\pi_{\alpha}\left(\alpha_{t+1} \mid \alpha_{t}\right)=\frac{1}{2} \delta(0)+\frac{1}{2} \delta(1)
$$

In the spirit of [6], a possible choice for the importance function of $z_{t}$ is its propagation density

$$
\pi_{z}\left(z_{t+1} \mid z_{t}, x_{t}, y_{t}, \alpha_{t}\right)=p\left(z_{t+1} \mid z_{t}\right)
$$

or, in the case of discarding the parameter $\alpha_{t}$, we may choose

$$
\pi_{z}\left(z_{t+1} \mid z_{t}, x_{t}, y_{t}\right)=p^{\prime}\left(z_{t+1} \mid z_{t}\right)
$$

and so for the background process

$$
\pi_{x}\left(x_{t+1} \mid x_{t}, y_{t}\right)=p\left(x_{t+1} \mid x_{t}\right)
$$

For the joint importance density follows

$$
\pi_{x}\left(x_{t+1}, z_{t+1} \mid x_{t}, z_{t}, y_{t}\right)=p\left(x_{t+1} \mid x_{t}\right) p^{\prime}\left(z_{t+1} \mid z_{t}\right) .
$$

The importance densities may have higher variances then their corresponding propagation densities in order to 'capture' the additional uncertainty influenced from the observation model. The importance function of the parameters $\theta_{t}$ is highly dependent on the model dynamics and should be chosen accordingly. 


\subsection{Detection}

The indicator $I_{t}$ for the event is easily calculated by counting the number $Z$ of exact zeros over all $N$ particles at time step $t$, i.e.

$$
Z_{t}=\sum_{n=1}^{N} \delta_{z_{n, t}},
$$

where $\delta_{z_{n, t}}$ is the so called Kronecker-Delta, the discrete version of the Dirac substitution, with the property

$$
\delta_{z_{n, t}}= \begin{cases}0 & z_{n, t} \neq 0 \\ 1 & z_{n, t}=0\end{cases}
$$

Having calculated the number $Z_{t}$ of exact zeros, the event indicator $I_{t}$ is calculated by

$$
I_{t}=\left\{\begin{array}{lc}
0 & \frac{Z_{t}}{N}<\frac{1}{2} \\
1 & \text { else. }
\end{array}\right.
$$

\section{EXPERIMENTS}

\subsection{Setup}

For the background process, we use the following state propagation function

$$
x_{t+1}=12+0.5 x_{t} \sin (t / 5)+\mathcal{N}\left(0, \sigma_{x}^{2}\right)
$$

and the following observation function

$$
y_{t}=0.5 x_{t}^{2}-2+\mathcal{N}\left(0, \sigma_{o}^{2}\right),
$$

where $\mathcal{N}\left(m, \sigma^{2}\right)$ denotes a normal probability density function with mean $m$ and variance $\sigma^{2}$. The resulting state propagation function including the superpositional AR(1) process $z_{t}$ is given by

$$
x_{t+1}=12+0.5 x_{t} \sin (t / 5)+\mathcal{N}\left(0, \sigma_{x}^{2}\right)+z_{t+1},
$$

where the propagation of the $\mathrm{AR}(1)$ process is given by

$$
z_{t+1}=a z_{t}+\mathcal{N}\left(0, \sigma_{z}^{2}\right) .
$$

The extended state vector is determined by

$$
s_{t}=\left(x_{t}, z_{t}\right) .
$$

The importance functions for the states $x_{t}, z_{t}$ are chosen as

$$
\begin{aligned}
\pi\left(x_{t+1} \mid x_{n, t}, y_{1: t+1}\right) & =p\left(x_{t+1} \mid x_{n, t}\right) \\
\pi\left(z_{t+1} \mid z_{n, t}, y_{1: t+1}\right) & =0.5 \delta(0)+0.5 \mathcal{N}\left(a z_{n, t}, \sigma_{z}^{2}\right),
\end{aligned}
$$

with variances of

$$
\sigma_{\pi(x)}^{2}=6 \sigma_{x}^{2}, \sigma_{\pi(z)}^{2}=6 \sigma_{z}^{2} .
$$

The compound importance function is then defined by

$$
\begin{aligned}
& \pi\left(x_{t+1}, z_{t+1} \mid x_{n, t}, z_{n, t}, y_{1: t+1}\right) \\
= & \pi\left(x_{t+1} \mid x_{n, t}, y_{1: t+1}\right) \pi\left(z_{t+1} \mid z_{n, t}, y_{1: t+1}\right) \\
= & p\left(x_{t+1} \mid x_{n, t}\right)\left(0.5 \delta(0)+0.5 \mathcal{N}\left(a z_{n, t}, \sigma_{z}^{2}\right) .\right.
\end{aligned}
$$

It is obvious that the detection success probability depends on the variances $\sigma_{x}^{2}$ and $\sigma_{z}^{2}$. With different values for $\sigma_{x}^{2}$, an observation noise of $\sigma_{o}^{2}=0.001$ and an AR(1) process noise of $\sigma_{z}^{2}=0.2$, we performed simulations of the hidden states $x_{t}$ and $z_{t}$.

The event was activated within $T_{E}=[50,70[$. We calculated the detection rates, including the false positive alarm probability $e^{+}$, and false negative alarm probability $e^{-}$by repeating the state sequence estimations 50 times each. The number of particles was set to $N=500$.

\subsection{Results}

Figures 1, 2, 3 and 4 show the results of the filter estimates vs. true values of both the $x_{t}$ and the $z_{t}$ signals and the event detection indicator bars for the state-variances of $\sigma_{x}^{2}=10^{-2}$, $\sigma_{x}^{2}=10^{-3}, \sigma_{x}^{2}=10^{-4}$ and $\sigma_{x}^{2}=10^{-5}$. It is known from detection theory, that the success of correct detection depends on the noise of the signals. As expected, the detection error decreases for smaller variances of the noise of the $x$-signal. The detection errors are shown in table 1 .
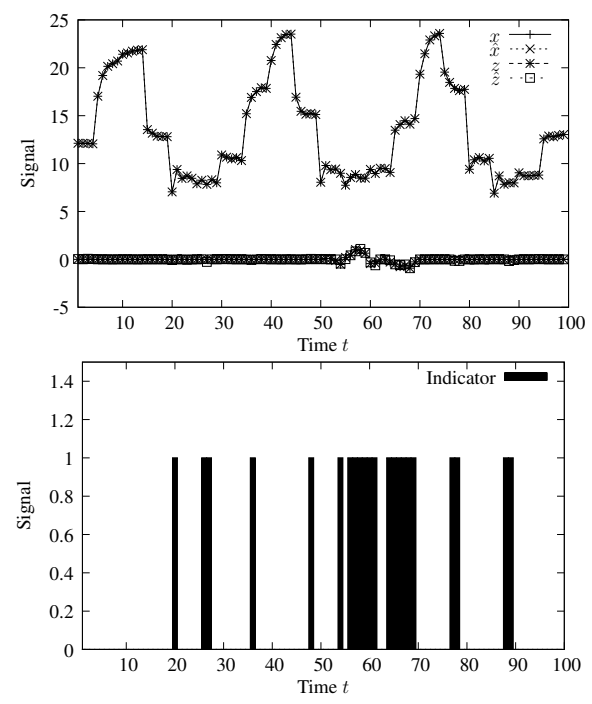

Fig. 1. True and estimated signals $x_{t}, z_{t}$ at $\sigma_{z}^{2}=2 \cdot 10^{-1}, \sigma_{x}^{2}=10^{-2}$

\section{CONCLUSIONS}

In case where events can be described by superpositional stochastic processes and the state propagation densities, a.k.a. the 'models' are known, the proposed framework can be used for online seperation and detection of 2 or more simultaneous events. Results can be further improved when the minimum activation time interval of the event signal is known and 

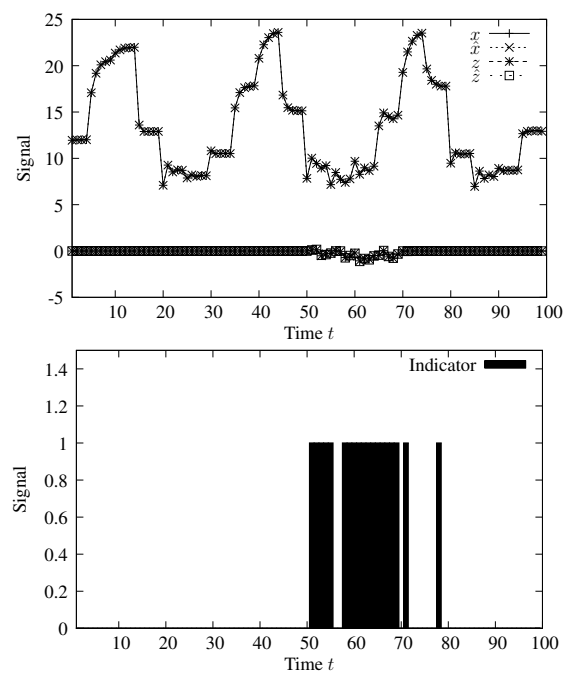

Fig. 2. True and estimated signals $x_{t}, z_{t}$ at $\sigma_{z}^{2}=2 \cdot 10^{-1}, \sigma_{x}^{2}=10^{-3}$
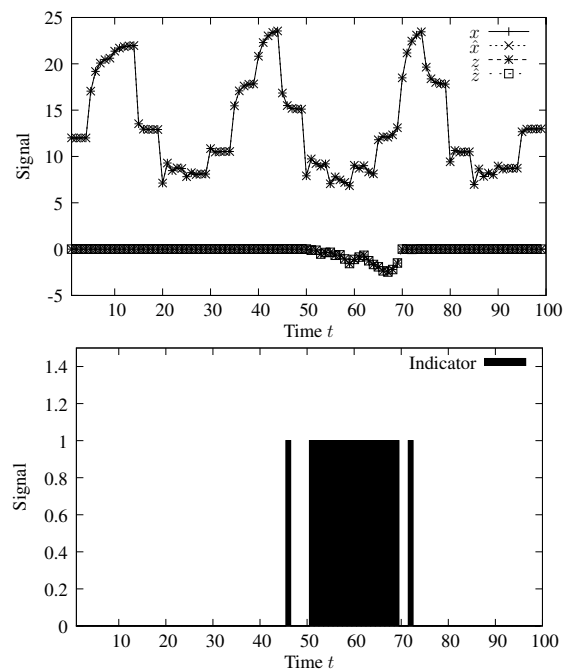

Fig. 3. True and estimated signals $x_{t}, z_{t}$ at $\sigma_{z}^{2}=2 \cdot 10^{-1}, \sigma_{x}^{2}=10^{-4}$

\begin{tabular}{|c|c||c|c|}
\hline$\sigma_{x}^{2}$ & $\sigma_{z}^{2}$ & $e^{+}$ & $e^{-}$ \\
\hline \hline $10^{-5}$ & $2 \cdot 10^{-1}$ & 0.0057 & 0.061 \\
$10^{-4}$ & $2 \cdot 10^{-1}$ & 0.0252 & 0.074 \\
$10^{-3}$ & $2 \cdot 10^{-1}$ & 0.0582 & 0.105 \\
$10^{-2}$ & $2 \cdot 10^{-1}$ & 0.1115 & 0.186 \\
\hline
\end{tabular}

Table 1. Event detection false positive alarm probabilities and false negative alarm probabilities for several background process noise variances

covers more than 1 sample. In this case, a modified event indicator would depend on the whole time interval and decide accordingly.
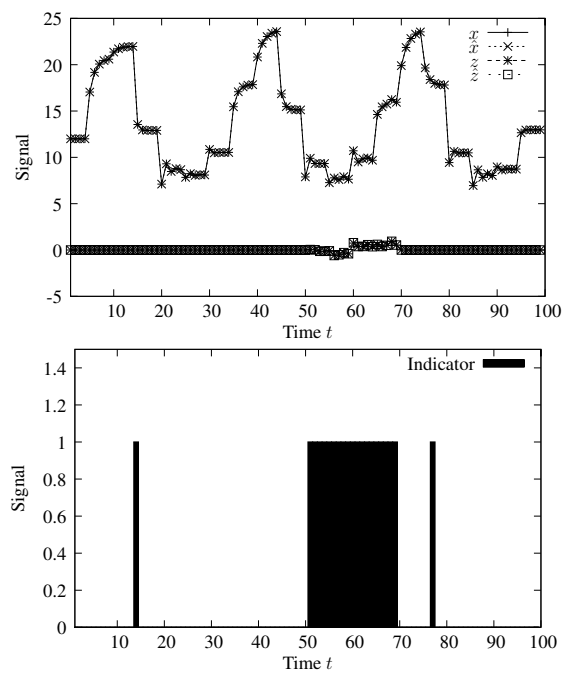

Fig. 4. True and estimated signals $x_{t}, z_{t}$ at $\sigma_{z}^{2}=2 \cdot 10^{-1}, \sigma_{x}^{2}=10^{-5}$

\section{REFERENCES}

[1] C. Andrieu, A. Doucet, S. S. Singh, and V. B. Tadic. Particle methods for change detection, system identification, and control, 2004.

[2] P. Del Moral. Feynman-Kac Formulae: Genealogical and Interacting Particle Systems with Applications. Springer, 2004.

[3] A. Doucet. On sequential simulation-based methods for bayesian filtering, 1998. Technical Report CUED/FINFENG/TR.310.

[4] A. Doucet, J. F. G. de Freitas, and N. J. Gordon. Sequential Monte Carlo Methods in Practice. Springer, 2001.

[5] P. Fearnhead. Sequential Monte Carlo methods in filter theory. Disseration, University of Oxford, 1998.

[6] M. Isard and A. Blake. Condensation - conditional density propagation for visual tracking. Int. J. Computer Vision, 29:528, 1998.

[7] M. Lavielle and E. Lebarbier. An application of mcmc methods for the multiple change-points problem. Signal Processing, 81(1):39-53, January 2001.

[8] J. S. Liu. Monte Carlo Strategies in Scientific Computing. Springer, 2001.

[9] S. Suparman, M. Doisy, Tourneret, and J.-Y. Changepoint detection using reversible jump mcmc methods. In IEEE International Conference on Acoustics, Speech and Signal Processing, volume 2, pages 1569-1572, 2002.

[10] J.-Y. Tourneret, M. Doisy, and M. Lavielle. Bayesian off-line detection of multiple change-points corrupted by multiplicative noise: application to sar image edge detection. Signal Process., 83(9):1871-1887, 2003.

[11] D. Zotkin, R. Duraiswami, and L. S. Davis. Multimodal 3-d tracking and event detection via the particle filter. In Workshop on Event Detection in Video, International Conference on Computer Vision, 2001. 